\title{
Total Number of Lymph Nodes in Oncologic Resections, Is There More to Be Found?
}

\author{
Kirsten J. de Burlet • Mari F. C. M. van den Hout • Hein Putter • \\ Vincent T. H. B. M. Smit • Henk H. Hartgrink
}

Received: 16 November 2014 / Accepted: 26 January 2015 / Published online: 18 February 2015

(C) 2015 The Author(s). This article is published with open access at Springerlink.com

\begin{abstract}
Pathologic staging of oncologic specimens includes the identification of the accurate lymph node status. Retrieving more lymph nodes leads to a more reliable N0 status in the TNM classification. The aim of this prospective study was to evaluate whether more lymph nodes can be retrieved from oncologic resection specimens when more time is invested in the search and if this contributes to a more reliable N-status in the individual patient. A total of 67 gastrointestinal oncologic specimens were reexamined for additional lymph nodes. The mean number of lymph nodes collected in the prospective group was compared against two retrospective groups, one before minima for lymph node counts were set (retrospective group 1) and one after (retrospective group 2). More lymph nodes were dissected per specimen in the prospective group (24.1 lymph nodes), compared to the retrospective group (14.3 lymph nodes, $P=<0.001$ ). During the study period, more patients were diagnosed as $\mathrm{pN}+$ compared to the two retrospective groups ( 62.7 vs. $47.8 \%$ respectively, $P=0.082)$. Significantly more lymph nodes can be found in oncologic specimens when more time is invested in the search. This will result in more accurate staging of the tumor.
\end{abstract}

Keywords Lymph node count $\cdot$ Staging $\cdot$ Oncologic resections $\cdot$ TNM classification

\section{Introduction}

Accurate pathologic staging of oncologic specimens includes the identification of lymph node metastases. Retrieving more lymph nodes leads to a more reliable $\mathrm{N}$ status, especially the pN0 status in the TNM classification, thereby improving the accuracy of 5-year prognosis. ${ }^{1-3}$ Moreover, the finding of even a single lymph node metastasis has, in some tumor types, major implications for adjuvant therapy.

K. J. de Burlet $(\bowtie) \cdot$ H. H. Hartgrink

Department of Surgery, K6-50, Leiden University Medical Center,

Albinusdreef 2, 2333 ZA Leiden, The Netherlands

e-mail: kjdeburlet@gmail.com

M. F. C. M. van den Hout • V. T. H. B. M. Smit

Department of Pathology, Leiden University Medical Center,

Leiden, The Netherlands

H. Putter

Department of Medical Statistics, Leiden University Medical Center, Leiden, The Netherlands
Concerning the number of lymph nodes that should be evaluated, oncologic guidelines like the American Joint Committee on Cancer (AJCC, www.cancerstaging.org) suggest more than 12 lymph nodes dissected per colorectal specimen and more than 15 lymph nodes for gastric cancer. $^{2-6}$ There are no guidelines with cutoff values for the extent of lymphadenectomy regarding esophagectomy or pancreaduodenectomy (Whipple) specimens.

Several studies have shown that if more lymph nodes are found in a pT1-3N0M0 cancer patient, it will lead to a longer 5 -year survival rate. This is reported for several types of cancer, like esophageal, stomach, colon, and rectal cancer. ${ }^{3-7}$ The most likely explanation for the observed association between increased number of negative lymph nodes and improved survival is incorrect staging of patients with missed positive lymph nodes due to the low total number of investigated lymph nodes. So, the reliability of the $\mathrm{pN} 0$ status will increase when more node negative lymph nodes are found..$^{5-11}$

An extended lymphadenectomy not only includes the enlarged lymph nodes. It is well known that severely enlarged lymph nodes, especially the nodes that have lost their ellipsoid contour, often contain tumor. However, lymph node metastases are not only found in large lymph nodes. In fact, several studies have shown that more than half of the retrieved lymph nodes containing tumor deposits are smaller than $5 \mathrm{~mm} .^{12^{-15}}$ 
Variables that may influence lymph node retrieval are potentially related to the patient, the surgeon, the pathologist, and neo-adjuvant regimens. Several studies have tried to investigate these variables in more detail. Literature suggests that the largest variation in the number of lymph nodes retrieved in surgical specimens is accounted for by differences between pathologists and not between surgeons. ${ }^{16-20}$ Still, there is an ongoing discussion between both, especially when small numbers of lymph nodes are encountered. The surgeon may feel that there are more lymph nodes to be found in the surgical specimen, while the pathologists may state that the surgeon's resection was not complete enough.

In an attempt to shed light on this discussion, both specialists agreed to participate in the study format as designed below.

\section{Materials and Methods}

Between November 2011 and June 2012, all specimens from the following surgical oncologic resections were prospectively collected in the Leiden University Medical Center: esophagus, stomach (partial and total), pancreas (pancreaticoduodenectomy according to Whipple), colon, and rectum. Excluded were specimens which, after microscopic evaluation, were diagnosed as benign or gastrointestinal stromal tumors (GIST).

All patients underwent a potentially curative resection, and all of them were preoperatively discussed in a multidisciplinary team with radiologists, oncologists, radiotherapists, surgeons, and pathologists. In these weekly meetings, decisions were made about the type of resection and, if relevant, (neo-)adjuvant treatment. Current protocol in the Leiden University Medical Center (based on national guidelines) recommends neo-adjuvant chemoradiation therapy (CRT) for esophageal tumors, neo-adjuvant chemotherapy (CT) for gastric cancer, and neo-adjuvant radiotherapy (RT) for T2-T4 rectal carcinomas. Neo-adjuvant therapy was renounced if a patient was unfit for the recommended treatment. Surgical techniques and protocols for (neo-)adjuvant treatment did not change during the study period.

Every specimen was routinely evaluated by the pathologist. This included a complete lymph node search of all the resected tissue by the pathology resident after overnight formalin fixation and microscopic evaluation of the lymph nodes by the resident and senior pathologist. During the study period, the mean time invested for this basic lymph node search was $20 \mathrm{~min}$ per specimen. Consequently, during the same week, a researcher $(\mathrm{KdB})$ searched for additional lymph nodes in the residual macroscopic tissue for exactly $20 \mathrm{~min}$. The number of potential lymph nodes retrieved was recorded every $5 \mathrm{~min}$ by the researcher. These included additional lymph nodes that were routinely processed and evaluated by the resident and senior pathologist and reported in the final pathology report. During the study period, mainly two pathology residents examined the specimens. One resident was involved from November until the end of December 2011 and the other from January until June 2012. On occasion, another resident pathologist substituted.

According to the Dutch rules for research for this study, no approval from the ethics committee was deemed necessary as only routine diagnostics were extended. Therefore, no informed consent was obtained from patients. For the researcher, all samples were handled in a coded fashion according to National Ethical Guidelines.

In order to evaluate the number of lymph nodes found in oncologic specimens in time, we retrospectively searched operation files for operated patients and matched them by organ and neo-adjuvant therapy to the prospective group.

Ultimately, retrospective group 1 consisted of surgical specimens from which lymph node data were collected between January and December 2008. Retrospective group 2 consisted of surgical specimens retrospectively collected between September 2010 and November 2011. These periods were deliberately chosen before and after 2009 because in that year, minima were set for the number of lymph nodes dissected in colon and stomach resections to improve accurate staging (AJCC). In this manner, we could also evaluate the development of lymph node counts under different protocols.

The database was statistically analyzed in SPSS (version 20). Numerical variables are summarized as mean (SD) or range. The differences between the retrospective and prospective group characteristics (age and gender) were assessed using the independent samples $t$ test for age and chi-square test for gender. Differences between groups with respect to number of lymph nodes found were assessed using the independent samples $t$ test (comparisons between retrospective 1 and 2 , between retrospective 2 and prospective A) and the paired samples $t$ test (comparison between prospective groups $\mathrm{A}$ and $\mathrm{B})$.

\section{Results}

The prospectively examined group consisted of a total of 67 oncologic specimens from patients operated in the Leiden University Medical Center. These specimens were categorized by six different types of resections performed between November 2011 and June 2012 (Table 1). The mean age of the patients was 66.3 years, and $57 \%$ of the patients were male. The mean age and gender did not differ significantly between the retrospective and prospective groups. As shown in Table 1, 25 patients received neo-adjuvant therapy. Twelve of them received neo-adjuvant chemoradiotherapy (CRT). Eleven were diagnosed with esophageal cancer and one with gastric cancer. The latter was preoperatively diagnosed as 
Table 1 Specimen characteristics

Esophagus $(n=11) \quad$ Partial gastric $(n=10) \quad$ Total gastric $(n=8) \quad$ Pancreas $(n=25) \quad$ Colon $(n=9) \quad \operatorname{Rectum}(n=4) \quad$ Total $(n=67)$

\begin{tabular}{|c|c|c|c|c|c|c|c|}
\hline \multicolumn{8}{|c|}{ Neo-adjuvant treatment, $n(\%)$} \\
\hline $\mathrm{CT}$ & $0(0 \%)$ & $5(50 \%)$ & $6(75 \%)$ & $0(0 \%)$ & $0(0 \%)$ & $0(0 \%)$ & $11(16.4 \%)$ \\
\hline RT & $0(0 \%)$ & $0(0 \%)$ & $0(0 \%)$ & $0(0 \%)$ & $0(0 \%)$ & $2(50 \%)$ & $2(3.0 \%)$ \\
\hline CRT & $11(100 \%)$ & $0(0 \%)$ & $1(12.5 \%)$ & $0(0 \%)$ & $0(0 \%)$ & $0(0 \%)$ & $12(17.9 \%)$ \\
\hline \multicolumn{8}{|c|}{ Tumor, $n(\%)$} \\
\hline $\mathrm{T} 1$ & $2(18.2 \%)$ & $1(10.0 \%)$ & $2(25.0 \%)$ & $4(15.4 \%)$ & $2(22.2 \%)$ & $0(0 \%)$ & $11(16.4 \%)$ \\
\hline $\mathrm{T} 2$ & $6(54.5 \%)$ & $1(10.0 \%)$ & $3(37.5 \%)$ & $5(20.0 \%)$ & $0(0 \%)$ & $2(50.0 \%)$ & $17(25.4 \%)$ \\
\hline $\mathrm{T} 3$ & $2(18.2 \%)$ & $7(70.0 \%)$ & $3(37.5 \%)$ & $16(64.0 \%)$ & $6(66.7 \%)$ & $2(50.0 \%)$ & $36(53.7 \%)$ \\
\hline $\mathrm{T} 4$ & $1(9.1 \%)$ & $1(10.0 \%)$ & $0(0 \%)$ & $0(0 \%)$ & $1(11.1 \%)$ & $0(0 \%)$ & $3(4.5 \%)$ \\
\hline \multicolumn{8}{|l|}{ Node, $n(\%)$} \\
\hline No & $3(27.3 \%)$ & $2(20.0 \%)$ & $4(50.0 \%)$ & $9(36.0 \%)$ & $4(44.4 \%)$ & $3(75.0 \%)$ & $25(32.8 \%)$ \\
\hline N1 & $6(54.5 \%)$ & $4(40.0 \%)$ & $2(25.0 \%)$ & $16(64.0 \%)$ & $4(44.4 \%)$ & $1(25.0 \%)$ & $33(49.3 \%)$ \\
\hline $\mathrm{N} 2$ & $2(18.2 \%)$ & $1(10.0 \%)$ & $2(25.0 \%)$ & $0(0 \%)$ & $1(11.1 \%)$ & $0(0 \%)$ & $6(9.0 \%)$ \\
\hline N3 & $0(0 \%)$ & $3(30.0 \%)$ & $0(0 \%)$ & $0(0 \%)$ & $0(0 \%)$ & $0(0 \%)$ & $3(4.5 \%)$ \\
\hline Total, $n(\%)$ & 11 & 10 & 8 & 25 & 9 & 4 & 67 \\
\hline
\end{tabular}

Neo-adjuvant treatment: $\mathrm{CT}=$ chemotherapy, $\mathrm{RT}=$ radiotherapy, and CRT $=$ chemotherapy and radiotherapy. Tumor and node according to TNM classification of the different specimens. Resection: R0 complete resection, R1 microscopic incomplete resection and R2 macroscopic incomplete resection

esophageal cancer, but after resection, the tumor proved to be of gastric origin. Eleven patients with a gastric tumor received neo-adjuvant chemotherapy (CT), and two patients with a rectal tumor received neo-adjuvant radiotherapy (RT) $(5 \times$ 5 Gy).

Tumor and node classification in this specimen cohort is shown in Table 1. Most of the resected specimens were pT2 or pT3 tumors $(79.3 \%)$. Twenty-five patients were classified as a pN0 tumor.

The mean number, standard deviation (SD), and range of lymph nodes dissected for each type of specimen are shown in Table 2. Lymph node count was significantly different between all groups. In retrospective group 1, before the protocol with minimal number of lymph nodes to be retrieved was implemented, fewer lymph nodes were collected $(P=$ $<0.001)$. Finding none or one lymph node in an oncologic specimen was reported more than once in this cohort. After minima were set (retrospective group 2), significantly more lymph nodes were evaluated $(P=0.001)$ and no pathology report includes less than five lymph nodes evaluated.

Table 3 shows the mean total lymph node count, the malignant lymph node count, and the percentage of malignant lymph nodes. No significant differences were found in lymph node counts between the 25 patients treated with neo-adjuvant therapy and the 42 patients without neo-adjuvant therapy. The mean lymph node count in the two patients who received neoadjuvant RT is lower compared to the other patients. These patients had a rectal tumor, and mean lymph nodes dissected for rectal specimens were overall lower in this cohort (see Table 1).
The mean number of lymph nodes retrieved did not differ between the levels of response to the given neo-adjuvant therapy. Patients with a complete or a good response in this cohort had no tumor-positive lymph nodes in the resected specimen.

pT4 tumors had a significant higher lymph node count compared to the pT1-3 tumors. The percentage of malignant lymph nodes in the specimens shows a gradual increase of the amount of tumor-positive nodes when the tumor grows in size.

Table 2 Number of lymph nodes found per specimen and group

\begin{tabular}{llll}
\hline & Retrospective 1 & Retrospective 2 & Prospective \\
\hline Esophagus & $11.3[4.2]$ & $14.3[5.0]$ & $24.1[7.4]^{*}$ \\
& $4-20$ & $8-23$ & $12-35$ \\
Partial gastric & $10.3[6.0]$ & $18.4[7.0]^{*}$ & $31.8[9.2]^{*}$ \\
& $0-18$ & $8-28$ & $18-42$ \\
Total gastric & $17.6[7.3]$ & $14.9[5.3]$ & $27.1[9.3]^{*}$ \\
& $6-27$ & $8-22$ & $16-44$ \\
Pancreas & $9.1[5.5]$ & $11.6[3.7]$ & $21.1[7.1]^{*}$ \\
& $0-20$ & $6-21$ & $9-36$ \\
Colon & $12.9[4.5]$ & $17.3[5.2]^{*}$ & $24.1[5.9]^{*}$ \\
& $5-19$ & $11-28$ & $14-31$ \\
Rectum & $6.0[5.0]$ & $12.8[5.9]$ & $18.0[6.4]$ \\
& $1-11$ & $5-19$ & $14-31$ \\
Total & $10.9[7.0]$ & $14.3[5.5]^{*}$ & $24.1[8.3]^{*}$ \\
& $0-27$ & $5-28$ & $9-44$ \\
\hline
\end{tabular}

Mean amount of lymph nodes found per group, [SD] and range

$* P=<0.05$, significant differences in mean lymph node count between the groups and per specimen (an independent sample $t$ test was used) 
Table 3 Mean lymph node count dependent of tumor size and neoadjuvant treatment

Total lymph Malignant Percentage node count lymph node malignant lymph (mean) count (mean) nodes (\%)

\begin{tabular}{|c|c|c|c|}
\hline \multicolumn{4}{|l|}{ Neo-adjuvant treatment } \\
\hline $\operatorname{Nil}(n=42)$ & 23.0 & 4.5 & 19.6 \\
\hline $\mathrm{CT}(n=10)$ & 30.8 & 3.6 & 11.7 \\
\hline $\mathrm{RT}(n=2)$ & 19.5 & 1.0 & 5.1 \\
\hline CRT $(n=13)$ & 23.3 & 2.8 & 12.0 \\
\hline \multicolumn{4}{|l|}{ Response } \\
\hline $\begin{array}{l}\text { No neo-adjuvant } \\
\text { treatment }(n=42)\end{array}$ & 23.0 & 4.3 & 18.7 \\
\hline No response $(n=3)$ & 25.7 & 1.7 & 6.6 \\
\hline Partial $(n=19)$ & 26.1 & 3.6 & 13.8 \\
\hline Good/complete $(n=3)$ & 25.7 & 0 & \\
\hline \multicolumn{4}{|l|}{ Tumor } \\
\hline $\mathrm{T} 1(n=11)$ & 23.4 & 1.2 & 5.1 \\
\hline $\mathrm{T} 2(n=17)$ & 24.3 & 2.8 & 11.5 \\
\hline $\mathrm{T} 3(n=36)$ & 23.7 & 4.7 & 19.8 \\
\hline $\mathrm{T} 4(n=3)$ & 30.0 & 11.0 & 36.7 \\
\hline \multicolumn{4}{|l|}{ Node } \\
\hline N0 $(n=25)$ & 24.1 & 0 & 0 \\
\hline $\mathrm{N} 1(n=33)$ & 23.6 & 4.0 & 16.9 \\
\hline $\mathrm{N} 2(n=6)$ & 23.8 & 8.0 & 33.6 \\
\hline $\mathrm{N} 3(n=3)$ & 29.7 & 25.0 & 84.2 \\
\hline Total $(n=67)$ & 24.1 & 3.8 & 15.8 \\
\hline
\end{tabular}

Neo-adjuvant treatment: $\mathrm{CT}=$ chemotherapy, $\mathrm{RT}=$ radiotherapy, and $\mathrm{CRT}=$ chemotherapy and radiotherapy. Tumor and node according to TNM classification of the different specimens. Resection: R0 complete resection, R1 microscopic incomplete resection, and R2 macroscopic incomplete resection

Table 4 shows the number of patients diagnosed as $\mathrm{N}+$ in the retrospective groups and the prospective group. More patients were diagnosed as $\mathrm{N}+$ in the prospective group (32 vs. $42, P=0.082$ ). No difference was found between the two retrospective groups. There is no clear correlation between lymph node positivity $(\mathrm{N}+)$ and tumor origin.

To evaluate the amount of dissected lymph nodes within the 20-min additive search, the cumulative mean number of

Table 4 Differences in N status of the TNM classification in the study groups

\begin{tabular}{llll}
\hline & Retrospective 1 & Retrospective 2 & Prospective \\
\hline N0 & $35(52.2 \%)$ & $35(52.2 \%)$ & $25(37.3 \%)$ \\
N+ & $32(47.8 \%)$ & $32(47.8 \%)$ & $42(62.7 \%)$ \\
Total & $67(100 \%)$ & $67(100 \%)$ & $67(100 \%)$ \\
\hline
\end{tabular}

In Table 3, the amounts and percentages of total patients diagnosed with $\mathrm{pN} 0$ or $\mathrm{pN}+$ per group are shown. More patients were diagnosed as $\mathrm{N}+$ in the prospective group, and this was not significant $(P=0.082)$ probable lymph nodes found per $5 \mathrm{~min}$ was calculated. Adding 5- or 10-min search time increased the number of retrieved lymph nodes by 12 and $20 \%$, respectively. Lymph node retrieval decreased logarithmically in time. After $20 \mathrm{~min}$, the cumulative mean number of extra lymph nodes retrieved was 6 ; this accounted for $25 \%$ of the total number of dissected lymph nodes.

\section{Discussion}

In this study, we found that when more time is invested in lymph node search, significantly more lymph nodes can be found in cancer resection specimens. Firstly, there is a clear improvement in lymph node retrieval after minima for the number of dissected lymph nodes per specimen were set in 2009. Secondly, we observed a significant difference between numbers of lymph nodes retrieved in the retrospective group 2 (after these minima were set) and the number of lymph nodes found in the prospective group. This leads to more patients diagnosed as $\mathrm{N}+$ in the prospective cohort compared to the two retrospective cohorts; however, no significant difference was found $(P=0.082)$.

Other variables that may influence the number of lymph nodes found are related to the surgeon and possible neoadjuvant therapy. If the surgical resection lacks sufficient lymphatic tissue, the pathologist will obtain fewer nodes. From studies on more extended lymph node dissections, we know that the surgical technique can make a great difference on the number of lymph nodes found. For gastric cancer, this was shown in a Dutch trial which showed an average of 17 lymph nodes found after a limited (D1) dissection and an average of 30 lymph nodes found after an extended (D2) dissection. ${ }^{21}$ In the current study, the same surgical resection techniques per specimen were used for all patients. Therefore, the surgical factor in the differences in lymph node retrieval found in this study is considered limited.

Some studies have found substantial variability between pathologists and the number of evaluated lymph nodes. Ostadi et al. ${ }^{17}$ evaluated factors which contributed to a difference in lymph node count in colorectal specimens. They concluded that most variation was accounted for by differences between pathologists. Evans et al. ${ }^{18}$ found no differences in lymph node harvest in colorectal cancer between individual surgeons but did find that there was a significant difference between reporting pathologists. Kuiper et al. ${ }^{19}$ reported that their laboratory technicians retrieved more lymph nodes than the pathologists, and Mekenkamp et al. ${ }^{20}$ found large differences in lymph node retrieval between various pathologists and between different laboratories. Based on the current study and the four studies cited above, it appears that the time invested in lymph node retrieval is the most crucial factor for adequate lymph node retrieval. ${ }^{17-20}$ 
From the pathologist's point of view, there is a consensus that neo-adjuvant CRT may result in the retrieval of fewer lymph nodes, especially when conventional manual lymph node dissections are used. ${ }^{2022}$ Mekenkamp et al. ${ }^{20}$ showed that after neo-adjuvant RT, fewer lymph nodes were retrieved in rectum specimens ( 6.9 vs. $8.5 ; P<0.0001)$. In esophageal cancer, the same effect of CRT to lymph node retrieval has been shown (6.9 vs. $8.6 ; P=0.026) .{ }^{23}$ RT is thought to decrease lymph node yield as a result of an immune response, rendering them undetectable because of RT-induced fibrosis. The shrinkage after RT makes lymph node retrieval more challenging. Nevertheless, in the above-mentioned studies, lymph node metastasis (and micro-metastasis) has been found in lymph nodes $<0.3 \mathrm{~cm}$. Thereby suggesting that, although lymph node evaluation is more challenging, it is crucial for accurate staging. ${ }^{24-26}$ In our study, there was no significant difference in lymph node counts between specimens treated with or without neo-adjuvant (C)RT. However, the groups were too small for accurate statistic analysis.

In order to facilitate large amounts of lymph node recovery, fat clearance techniques have been developed. Average lymph node counts over 50 have been reported when using these techniques. However, fat clearance techniques are not often used because they are time-consuming ( 1 to 5 weeks extra per specimen) and more expensive. ${ }^{25 \cdot 26}$

Comparing the number of patients diagnosed as $\mathrm{pN}+$ in our three groups shows an interesting result. The adjustment of guidelines did lead to an increased number of lymph nodes evaluated, but not to a change in percent of patients staged with $\mathrm{pN}+$ disease. In the prospective group, however, we observed a clear stage migration since more patients were diagnosed as $\mathrm{pN}+$ compared to the retrospective groups (62.7 vs. $47.8 \%$, respectively, $P=0.082$ ). The more extensive search in the prospective groups may have led to this stage migration phenomenon.

The estimated average search time for lymph node retrieval by the pathology resident was approximately $20 \mathrm{~min}$. The time for reexamination for additional lymph nodes was set at $20 \mathrm{~min}$ as a longer search seemed unfeasible for routine pathological practice. In the additional search, the largest number of extra lymph nodes was found during the first $5 \mathrm{~min}$, and this number decreased logarithmically in time. Adding 5- or 10min search time increased the number of retrieved lymph nodes by 12 and $20 \%$, respectively. Although data are insufficient to make firm conclusions, considering the estimated search time by the pathology resident, the optimal total time for lymph node retrieval may be around $30 \mathrm{~min}$, although this may differ between oncologic specimens and origin, depending on the amount of fatty tissue.

There are some limitations to our study. Firstly, the number of extra lymph nodes found by the researcher was registered every $5 \mathrm{~min}$, but the initial lymph node search by the pathology resident was not recorded in detail. Secondly, no data was collected on the outcome and the possible change in adjuvant treatment and prognosis. For certain tumor types, like pancreas carcinomas, the "stage migration" due to the extra dissected lymph nodes would not lead to a change in postoperative management. However, it will make a difference in prognosis, and therefore, it will still be relevant for the patient. For other tumor types, like colon cancer, it may alter adjuvant treatment.

Finally, the data from the two retrospective groups was collected via the electronic database of the hospital. This has its obvious retrospective data collection hazards, and for our study, specifically, there was no indication of the total time invested in lymph node search.

\section{Conclusion}

Significantly more lymph nodes can be found in oncologic specimens when more time for searching is invested. This will improve accurate staging of the patients and could have important implications for (adjuvant) therapy and prognosis. The optimal time for retrieval of lymph nodes may be around $30 \mathrm{~min}$, and the surplus value of retrieving decreases in time.

Acknowledgments The authors declare that no source of funding was used for this manuscript and no competing interests have been reported.

Open Access This article is distributed under the terms of the Creative Commons Attribution License which permits any use, distribution, and reproduction in any medium, provided the original author(s) and the source are credited.

\section{References}

1. Chang GJ, Rodriguez-Bigas MA, Skibber JM, Moyer VA. Lymph node evaluation and survival after curative resection of colon cancer: Systematic review. J Natl Cancer Inst 2007;99:433-441.

2. Le Voyer TE, Sigurdson ER, Hanlon AL, Mayer RJ, Macdonald JS, Catalano PJ, Haller DG. Colon cancer survival is associated with increasing number of lymph nodes analyzed: A secondary survey of intergroup trial INT-0089. J Clin Oncol 2003;21:2912-2919.

3. Swanson RS, Compton CC, Stewart AK, Bland KI. The prognosis of T3N0 colon cancer is dependent on the number of lymph nodes examined. Ann Surg Oncol 2003;10:65-71.

4. Cianchi F, Palomba A, Boddi V, Messerini L, Pucciani F, Perigli G, Bechi P, Cortesini C. Lymph node recovery from colorectal tumor specimens: Recommendation for a minimum number of lymph nodes to be examined. World J Surg 2002;26:384-389.

5. Greenstein AJ, Litle VR, Swanson SJ, Divino CM, Packer S, Wisnivesky JP. Effect of the number of lymph nodes sampled on postoperative survival of lymph node-negative esophageal cancer. Cancer 2008;112:1239-1246.

6. Yang HX, Xu Y, Fu JH, Wang JY, Lin P, Rong TH. An evaluation of the number of lymph nodes examined and survival for node-negative esophageal carcinoma: Data from china. Ann Surg Oncol 2010;17: 1901-1911.

7. Park IJ, Yu CS, Lim SB, Yoon YS, Kim CW, Kim TW, Kim JH, Kim JC. Prognostic implications of the number of retrieved lymph nodes 
of patients with rectal cancer treated with preoperative chemoradiotherapy. J Gastrointest Surg. 2014;18:1845-1851

8. Block MI. Transthoracic vs. transhiatal esophagectomy: Stage migration muddies the water. J Surg Oncol 2006;93:519-520.

9. Baxter NN, Morris AM, Rothenberger DA, Tepper JE. An evaluation of the relationship between lymph node number and staging in pT3 colon cancer using population-based data. Dis Colon Rectum 2010;53: 65-70

10. Wright FC, Law CHL, Berry S, SMITH AJ. Clinically Important Aspects of Lymph Node Assessment in Colon Cancer. J. Surg. Oncol 2009;99:248-255

11. Baxter NN. Is Lymph Node Count an Ideal Quality Indicator for Cancer Care? J. Surg. Oncol 2009;99:265-268

12. Rodriguez-Bigas MA, Maamoun S, Weber TK, Penetrante RB, Blumenson LE, Petrelli NJ. Clinical significance of colorectal cancer: Metastases in lymph nodes $<5 \mathrm{~mm}$ in size. Ann Surg Oncol 1996;3: 124-130.

13. Haboubi NY, Clark P, Kaftan SM, Schofield PF. The importance of combining xylene clearance and immunohistochemistry in the accurate staging of colorectal carcinoma. J R Soc Med 1992;85:386-388.

14. Monig SP, Baldus SE, Zirbes TK, Schroder W, Lindemann DG, Dienes HP, Holscher AH. Lymph node size and metastatic infiltration in colon cancer. Ann Surg Oncol 1999;6:579-581.

15. Schroder W, Baldus SE, Monig SP, Beckurts TK, Dienes HP, Holscher AH. Lymph node staging of esophageal squamous cell carcinoma in patients with and without neoadjuvant radiochemotherapy: Histomorphologic analysis. World J Surg 2002;26:584-587.

16. Herrera-Ornelas L, Justiniano J, Castillo N, Petrelli NJ, Stulc JP, Mittelman A. Metastases in small lymph nodes from colon cancer. Arch Surg 1987;122:1253-1256.

17. Ostadi MA, Harnish JL, Stegienko S, Urbach DR. Factors affecting the number of lymph nodes retrieved in colorectal cancer specimens. Surg Endosc 2007;21:2142-2146.

18. Evans MD, Barton K, Rees A, Stamatakis JD, Karandikar SS. The impact of surgeon and pathologist on lymph node retrieval in colorectal cancer and its impact on survival for patients with dukes' stage B disease. Colorectal Dis 2008; 10:157-164.

19. Kuijpers CC, van Slooten HJ, Schreurs WH, Moormann GR, Abtahi MA, Slappendel A, Cliteur V, van Diest PJ, Jiwa NM. Better retrieval of lymph nodes in colorectal resection specimens by pathologists' assistants. J Clin Pathol 2013;66:18-23.

20. Mekenkamp LJ, van Krieken JH, Marijnen CA, van de Velde CJ, Nagtegaal ID, Pathology Review Committee and the Co-operative Clinical Investigators. Lymph node retrieval in rectal cancer is dependent on many factors-the role of the tumor, the patient, the surgeon, the radiotherapist, and the pathologist. Am J Surg Pathol 2009;33: 1547-1553.

21. Hartgrink HH, van de Velde CJ, Putter H, Bonenkamp JJ, Klein Kranenbarg E, Songun I, Welvaart K, van Krieken JH, Meijer S, Plukker JT, van Elk PJ, Obertop H, Gouma DJ, van Lanschot JJ, Taat CW, de Graaf PW, von Meyenfeldt MF, Tilanus H, Sasako M. Extended lymph node dissection for gastric cancer: Who may benefit? final results of the randomized Dutch gastric cancer group trial. J Clin Oncol 2004;22:2069-2077.

22. Miller ED, Robb BW, Cummings OW, Johnstone PA. The effects of preoperative chemoradiotherapy on lymph node sampling in rectal cancer. Dis Colon Rectum 2012;55:1002-1007.

23. Chao YK, Liu HP, Hsieh MJ, Wu YC, Liu YH, Yeh $\mathrm{CH}$, Chang HK, Tseng CK. Lymph node dissection after chemoradiation in esophageal cancer: A subgroup analysis of patients with and without pathological response. Ann Surg Oncol 2012;19:3500-3505.

24. Sprenger T, Rothe H, Homayounfar K, Beissbarth T, Ghadimi BM, Becker H, Liersch T. Preoperative chemoradiotherapy does not necessarily reduce lymph node retrieval in rectal cancer specimensresults from a prospective evaluation with extensive pathological work-up. J Gastrointest Surg 2010;14:96-103.

25. Gehoff A, Basten O, Sprenger T, Conradi LC, Bismarck C, Bandorski D, Merkelbach-Bruse S, Schneider-Stock R, Stoehr R, Wirtz RM, Kitz J, Muller A, Hartmann A, Becker H, Ghadimi BM, Liersch T, Ruschoff J. Optimal lymph node harvest in rectal cancer (UICC stages II and III) after preoperative 5-FU-based radiochemotherapy. acetone compression is a new and highly efficient method. Am J Surg Pathol 2012;36:202-213.

26. Wang H, Safar B, Wexner SD, Denoya P, Berho M. The clinical significance of fat clearance lymph node harvest for invasive rectal adenocarcinoma following neoadjuvant therapy. Dis Colon Rectum 2009;52:1767-1773. 